\title{
Interactions of Microplastics with Persistent Organic Pollutants and the Ecotoxicological Effects: A Review
}

\author{
Kuok Ho Daniel Tang \\ Environmental Science Program, Division of Science and Technology, BNU-HKBU United International College, 2000 \\ Jintong Road, Tangjiawan, Zhuhai, GD 519087, China \\ *Correspondence: daniel.tangkh@yahoo.com
}

SUBMITTED: 25 May 2021; REVISED: 16 June 2021; ACCEPTED: 18 June 2021

\begin{abstract}
With the increasing presence of microplastics and persistent organic pollutants (POPs), it is crucial to understand the interactions between the two emerging environmental pollutants and their ecotoxicological risks. This paper reviews more than 50 relevant scholarly papers published mainly in the past 10 years. It shows that the sorption of POPs to microplastics is affected by environmental factors and the properties of microplastics. The environmental factors comprise salinity, $\mathrm{pH}$, natural organic matters and temperature. The properties of microplastics include degree of aging, molecular weight, size, shape, density, crystallinity, polymer type and color. The two factors are interconnected through weathering and weatherability of microplastics, where properties of microplastics, hence their interactions with POPs would be modified by environmental factors. Microplastics are potential vectors of POPs due to their ability to sorb and concentrate POPs. However, the studies reviewed showed the impacts to be low or insignificant and the sorbed POPs do not demonstrate significantly high accumulation, bioavailability and toxicity. In some literature, it has been reported that microplastics might reduce POPs in an organism. Due to limited studies and opposing views, there is a need to conduct more studies involving diverse POPs and microplastics under multiple conditions to provide a more holistic understanding on this subject.
\end{abstract}

Keywords: Bioaccumulation, ecological, microplastics, persistent organic pollutants, sorption, toxicity

\section{Introduction}

Microplastics have been subjected to extensive study due to their prevalence in the environment. Microplastics have been found in nearly all compartments of the environment, ranging from marine and freshwater to soil and air [1]. The prevalence of microplastics is associated with the increasing use of plastics often due their versatility. Plastics are inexpensive, light-weighted, strong and they can be modified to impart specific properties such as chemical- and heat-resistance. Plastics have been used in a wide array of sectors such as aerospace, construction, electrical and electronic, packaging as well as medical and healthcare, to name a few [2]. 
Plastics enter the oceans at a rate of approximately 8 million pieces per day currently and 381 million tonnes of plastic waste is generated yearly. Single use plastics constitute about half of the plastic waste produced [3]. These plastic items degrade over time due to complex interactions with multiple environmental factors, producing microplastics with sizes of $5 \mathrm{~mm}$ and less [1]. As the breakdown of plastics usually occurs over a long time, microplastics tend to stay in the environment where they are transported, dispersed, distributed, deposited or further degraded [4]. Microplastics could also be contributed by the use of microbeads in personal care products and microfibers from the washing of synthetic textiles. These microplastics, also called the primary microplastics, undergo the same fate in the environment as the secondary microplastics formed from the degradation of larger plastics [5].

These microplastics could enter the food chains where bioaccumulation and biomagnification take place. They could provide the surface for adsorption of organic substances, including the persistent organic pollutants (POPs), thus, complicating the environmental pollution caused by microplastics alone [6]. POPs are organic compounds that are not readily degraded by physical, chemical and biological processes in the environment [7]. They are common ingredients for pesticides, solvents and pharmaceuticals. They tend to bioaccumulate, leading to potential ecotoxicological effects [7]. Besides, various additives are added during the manufacturing of plastics to achieve certain desirable properties. These additives could leach into the environment and add to the load of pollutants [8]. It is also possible for the desorption of organic substances adsorbed by microplastics to take place when the condition becomes suitable, for instance in a living receptor [8].

While microplastics have been shown to undergo such complex interactions in the environment with other pollutants, studies in this area are not as extensive as those related to the prevalence of microplastics. Studies on the interactions of microplastics with POPs are even less. Multiple studies focus on the interactions of microplastics with antibiotics and heavy metals. $\mathrm{Yu}$ et al. investigated the adsorption behaviors of tetracycline on different types and sizes of microplastics [9]. Guo and Wang investigated the sorption behaviors of sulfamethoxazole (SMX), sulfamethazine (SMT) and cephalosporin C (CEP-C) onto polystyrene (PS) and polyethylene (PE) microplastics which had undergone natural aging [10]. The study showed a predominantly linear isotherm governing sorption in freshwater with $\mathrm{K}_{\mathrm{d}}$ values between $0.0236 \mathrm{~L} / \mathrm{g}$ and $0.0383 \mathrm{~L} / \mathrm{g}$ [10]. Zhu et al. examined the prevalence and fate of microplastics in edible oysters and found microplastics to adsorb trace metals, potentially causing co-toxicity [11]. Fred-Ahmadu et al. reviewed the factors affecting sorption of pollutants on microplastics which include surface weathering, chemical properties of pollutants and degree of crystallinity of microplastics. The review did not aim to specifically examine how microplastics interact with POPs [12]. Having analyzed the data of published journal articles, Wang et al. found microplastics collected from large cities had higher concentrations of polychlorinated biphenyls (PCBs), dichlorodiphenyltrichloroethane (DDT) and hexachlorocyclohexane $(\mathrm{HCH})$ in comparison to those from small cities, rural precincts, and remote islands [13]. The review also highlighted variation of the concentrations of polycyclic aromatic hydrocarbons (PAHs) with depths of beach sediments. All substances reported in the review are POPs. It leans towards the spatial and temporal variations in the amounts of POPs sorbed by the microplastics as well as the sorption-affecting factors [13].

Liu et al. studied the sorption characteristics of diethyl phthalate (DEP) and dibutyl phthalate (DBP) onto polyvinyl chloride (PVC), PE and PS. This study revealed hydrophobic 
interaction as the main governing sorption mechanism and the degree of sorption was the highest for PS, followed by PE and PVC. It is experimental in nature, focusing on two common POPs [14]. A recent review by Menéndez-Pedriza and Jaumot focuses on the factors influencing the sorption behaviors of microplastics and the circumstances in which microplastics could facilitate the bioaccumulation and transfer of chemicals. The review does not confer special attention to POPs [15]. Similarly, a review of how organic pollutants interact with microplastics by $\mathrm{Fu}$ et al. takes on a general approach, looking into the mechanisms organic pollutants adsorb on microplastics and the determinants of their adsorption capacity [16].

With POPs receiving increasing attention due to their potential to remain in the environment, get transported to places they are not known to have been produced, as well as to enter the food chains, it is crucial to understand the interactions of POPs with the now ubiquitous microplastics and their combined toxicity. This article aims to systematically review the interaction mechanisms of POPs with microplastics and the resulting ecological risks.

\section{Methods}

Literature review was conducted on scholarly articles comprising journal and conference articles published predominantly in the past 10 years [17-18]. The articles were sourced from three main journal databases, namely Scopus, Web of Science and Elsevier. The keywords used in the literature search included POPs, microplastics, sorption, adsorption, interactions, toxicity, bioavailability and accumulation. More than 50 articles were reviewed.

\section{Discussion}

\subsection{Sorption of Organic Compounds by Microplastics}

Microplastics are known to provide the surface for sorption of an array of organic compounds. Sorption is fundamentally a combination of absorption and adsorption [19-20]. Unlike adsorption involving the attachment of molecules on the surface of a sorbent through forces such as Van-der-Waals and covalent bonds, absorption involves dissolution and retention of molecules within a sorbent through week Van-der-Waals forces [21]. Sorption can be concentration-dependent with absorption predominating at high concentrations of organic contaminants and adsorption predominating at low concentrations of organic contaminants [21]. Sorption is also affected by other factors such as properties of sorbents and sorbates.

The concern for the ability of microplastics to concentrate organic pollutants in the environment stems from the facts that microplastics and the pollutants are hydrophobic and microplastics provide large surface area for sorption of the pollutants [22-23]. Consequently, POPs as a subset of organic contaminants, which are commonly hydrophobic, tend to interact with nonpolar particles in the environment, such as microplastics [21]. The concentrations of organic compounds sorbed by microplastics could be six orders of magnitude higher compared to those in seawater [24].

\subsection{Factors Influencing Sorption of POPs by Microplastics}

Sorption of POPs by microplastics is influenced by numerous factors which are largely categorized as environmental factors and the inherent properties of microplastics. The 
environmental factors include salinity, $\mathrm{pH}$, natural organic matter, temperature and ionic strength while the properties of microplastics influencing sorption encompass size, shape, density, color, polymer type, crystallinity, molecular weight and degree of aging (see Figure 1) [25]. The sorption of POPs to microplastics is predominantly through hydrophobic interaction where hydrophobic substances, in this case POPs and microplastics, tend to come together in an aqueous environment. This is demonstrated by the linear isotherm between POPs and microplastics in a few studies [13-16]. For polar POPs, their interactions with microplastics could be partially affected electrostatic force. Taking the anionic perfluorooctanesulfonate for instance, the sulfonate group on the compound may hinder its sorption to PS due to electrostatic repulsion with the negative surface of PS [16]. In the case of perfluorooctanesulfonamide, a nonionic POP, it has higher sorption to PS compared to perfluorooctanesulfonate but its sorption to PS is lower than PE probably because of benzene rings in PS which obstruct bond rotation and occupy the free space between chains [16]. The interactions between POPs and microplastics are therefore highly variable and this review takes on a general approach to examine the overarching factors governing such interactions without narrowing down on specific POPs.

\subsubsection{Environmental Factors Affecting POPs Sorption}

Bakir et al. found desorption of POPs to increase in conditions with low $\mathrm{pH}$ and high temperature [26]. Variations in the contents of natural organic matter have been reported to affect sorption behaviors and dissolved organic matter seem to affect the solubility of hydrophobic POPs. Organic matters may promote sorption of POPs through hydrophobic interaction [21]. Liu et al., however, revealed that solution $\mathrm{pH}$ and natural organic matter did not substantially alter the sorption of phthalate esters to microplastics [14]. This implies that the environmental effects on sorption behaviors could be POPs-specific. In their study, phthalate esters were found to demonstrate better sorption to microplastics at higher salinity due to salting-out effect [14]. Salinity influences the agglomeration of microplastic particles, hence changes their sizes and surface areas. According to Velzeboer et al., higher sorption of contaminants by microplastics was observed at higher salinity which is posited to increase partitioning of microplastics [27]. On the contrary, Bakir et al. reported that increasing salinity did not significantly affect the sorption of phenanthrene to microplastics, while it decreased the sorption of DDT to microplastics marginally [26]. However, ionic strength is not expected to significantly affect the sorption of POPs by non-polar microplastics though it could alter solubility of POPs in water [19]. For certain polar POPs, for instance perfluorooctanesulfonate, higher ionic strength and lower $\mathrm{pH}$ were reported to facilitate its sorption to microplastics [13].

\subsubsection{Effects of Microplastics Weathering and Weatherability on POPs Sorption}

Weathering of microplastics has an important role in their interactions with POPs. Microplastics undergo time-dependent weathering in the environment caused predominantly by photo-oxidation, thermo-oxidation and biodegradation [28]. Intrinsic weatherability of microplastics also determines the extent of weathering experienced by microplastics. Weathering often alters their polymeric properties, hence sorption dynamics [29]. It links the environmental factors to the intrinsic properties of microplastics. Once in the environment, microplastics could undergo chemical changes. Oxidation of their surface might introduce new 
oxygen-containing functional groups which interfere with existing sorption patterns and permit sorption of new organic compounds [30]. Microplastics could also anchor biofilm, thus, exhibiting enhanced sorption in comparison to unaltered microplastics [30].

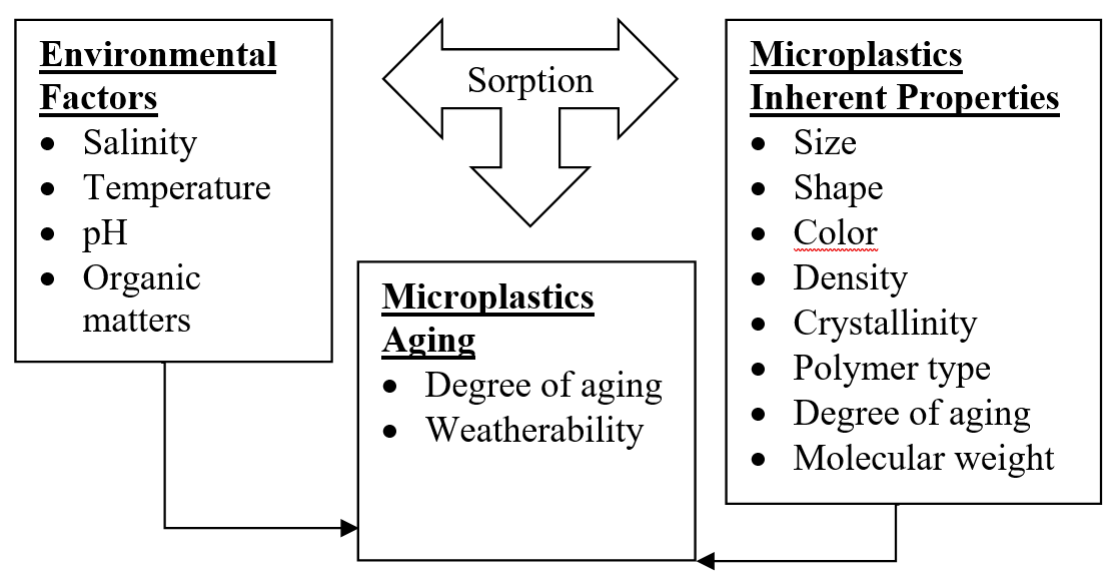

Figure 1. Factors affecting POPs sorption to microplastics

\subsubsection{Effects of Microplastics Properties on POPs Sorption}

Microplastics are physically and chemically diverse. Frias et al. found PAHs, PCBs and DDT to be present in all microplastic pellets sampled from two Portuguese beaches and the levels were higher in black and aged pallets [31]. This implies the potential influences of microplastics properties and aging on POPs sorption. Besides, colors of microplastics have been associated with degree of weathering and the molecular weights of PAHs attached where lighter pellets were reported to contained more PAHs of lower molecular weights and darker pellets contained PAHs of higher molecular weights [32]. Nonetheless, Müller et al. did not observe significant correlation between degree of aging and sorption of fuel aromatics by microplastics [33]. As with other polymers, microplastics generally possess crystalline and amorphous zones with the former consisting of closely packed polymer chains and the latter comprise loosely packed chains with voids [34]. POPs have higher tendency to sorb to the amorphous zone than the crystalline zone [35]. PS, which is highly crystalline is a poorer sorbent of POPs compared to PE and PP which have low crystallinity [36]. It is noteworthy that the drivers for sorption of POPs to microplastics often work in tandem. For instance, PE is an efficient sorbent attributed to its high surface area and low crystallinity.

In addition, the amorphous zone of a polymer has been differentiated into glassy or rubbery based on the glass transition temperature. A glassy amorphous zone has higher density and cross-linking, but does not crystallize. This region accounts for stronger adsorption and lower desorption of organic compounds [29]. PVC and PS are two examples of polymers containing glassy amorphous zones, thus conferring them high adsorptive capacity [37]. The rubbery amorphous zone, however, has higher diffusivity and permeability due to the presence of more voids. Therefore, polymers with predominantly rubbery amorphous zones such as PE and PP tend to absorb organic contaminants [36-37]. These polymers have been found to demonstrate greater ability to sorb and concentrate PCBs and PAHs compared to glassy polymers like PET and PVC [36]. Several studies also concur that among PE, PP and PVC which are most abundant in the environment, PE has the highest capacity to sorb and concentrate organic pollutants $[30,32,38]$. 
Sizes of microplastics determine their sorption capacity. With decreasing particle sizes, the interaction between microplastics and POPs increases. Velzeboer et al. reported that the sorption of PCBs to nano-PS was 1 to 2 orders of magnitude higher than micro-PE, while the sorption of PCBs to nanoparticles, namely multiwalled carbon nanotubes and fullerene was 3 to 4 orders of magnitude higher than micro-PE [27]. The sorption capacity of micro-PE was comparable to sediment organic matter. The higher sorption of nano-PS than micro-PE was attributed to higher aromaticity and surface area [27].

\subsection{Microplastics as Carriers of POPs}

POPs sorbed to microplastics can enter the food chain, resulting in ecotoxicity [39]. Due to their resemblance to food, microplastics have been reported to be ingested by various organisms, causing the entry of sorbed POPs into their digestive systems and circulatory systems subsequently. POPs are lipid-soluble and it is very likely for the ingested POPs to enter the fat tissues where they would accumulate. Microplastics containing POPs could also come into contact with the external and internal surfaces of an organism [39]. In aquatic environment, POPs may undergo desorption after which the contaminated water could be drawn in by aquatic organisms [21]. The levels of water contamination due to desorption of POPs are subject to factors such as diffusion and partitioning. Upon entering an organism, microplastics containing POPs might encounter conditions such as favorable $\mathrm{pH}$ of digestive fluids which enhance POPs desorption. Kärrman et al. found desorption of organic contaminants from plastics to be facilitated by the presence of surfactants and organic matter [40]. Bioaccumulation of POPs due to ingestion of microplastics has given rise to new concerns on food safety, particularly among mollusks which are filter feeders exhibiting greater tendency to bioaccumulate pollutants [41-42].

Diepens and Koelmans conducted a simulation of the transfer of microplastics, PAHs and PCBs in food webs and revealed higher biomagnification of PAHs upon co-ingestion of microplastics which reduced the availability of PAHs for metabolization [43]. This was not observed for PCBs as biomagnification of PCBs from regular food was more prevalent. The simulation showed a small effect of microplastics on accumulation of POPs and it could present a significantly different scenario from the actual situation [43]. Another study by Kleinteich et al. which exposed natural bacterial communities to microplastics with PAHs sorbed found sorption reduced bioavailability of PAHs and could potentially decrease POPs in aquatic environment [44]. Pittura et al. also tested how the presence of microplastics altered bioavailability of PAHs, specifically in Mytilus galloprovincialis (Mediterranean mussel) and unveiled marginally elevated cellular toxicity upon short-term exposure due to microplastics. It underscored a likely low ecotoxicological risk associated with microplastics which is not negligible [45]. Magara et al. found co-exposure of Mytilus edulis (blue mussel) to micro-PE and fluoranthene did not lead to observable additive or synergistic effects. Their study showed exposure to micro-PE alone was able to trigger oxidative stress [46].

Batel et al. studied the accumulation of benzo[a] pyrene (BaP), a kind of PAHs sorbed to microplastics in the gills and embryos of zebrafish. The study demonstrated that microplastics were constantly removed from the filaments of the adult zebrafish gills and this implies a lack of significant accumulation. The zebrafish egg chorions, however, accumulated the microplastics, particularly the heavier fraction, to a larger extent. Upon exposure to BaP-sorbed microplastics, the level of $\mathrm{BaP}$ was not sufficiently high to induce morphological change in the 
fish embryo, in contrary to the $\mathrm{BaP}$ in water [47]. Furthermore, Grigorakis and Drouillard fed Carassius auratus (goldfish) with diet containing PCBs-sorbed microplastics and examined the assimilation efficiencies. They found microplastics and the sorbed PCBs were of low bioavailability and posited that microplastics do not pose a significant concern for bioaccumulation of POPs by fish in aquatic environment [48]. A similar study by Devriese et al. examining the bioaccumulation of PCBs in the body parts of Nephrops norvegicus (Norway lobster) from PCBs-sorbed microplastics also pointed to insignificant bioaccumulation of PCBs after ingestion of the spiked micro-PE by the lobsters. The tail tissues of the organisms showed minimal uptake of PCBs. Besides, a 3-week ingestion of microplastics did not elicit noticeable effect on the nutritional state of the lobsters [49].

The studies above show variable results on the bioaccumulation, bioavailability and toxicity of POPs sorbed to microplastics. Nonetheless, they seem to point to low to insignificant toxicity, bioavailability and bioaccumulation of few POPs, namely PAHs, BaP and PCBs, where microplastics in certain instances actually reduced the bioavailability of PAHs. In comparison to POPs in the natural environment, biomagnification of POPs associated with microplastics is less prevalent. There seems to be a consensus among the studies that most of the toxic effects observed were due to microplastics themselves rather than the POPs sorbed. Ziccardi et al. were of the opinion that microplastics are unlikely to give rise to significant ecological risk though they have strong affinity towards POPs [25]. On the same note, Scopetani et al. commented on the roles of microplastics as carriers of POPs to biota as well as removers of POPs from their surroundings. They showed a balanced positive and negative impacts of microplastics through transfer to and reduction of POPs from an amphipod, which led them to conclude that microplastics and their associated pollutants have limited impacts on biota [50](Figure 2). Pannetier et al. pointed to the contrary when a few of their environmental microplastics samples induced toxicity in fish cell line, particularly modified ethoxyresorufino-deethylase (EROD) activities and DNA damage. These samples showed high concentration of PAHs and DDT [51].

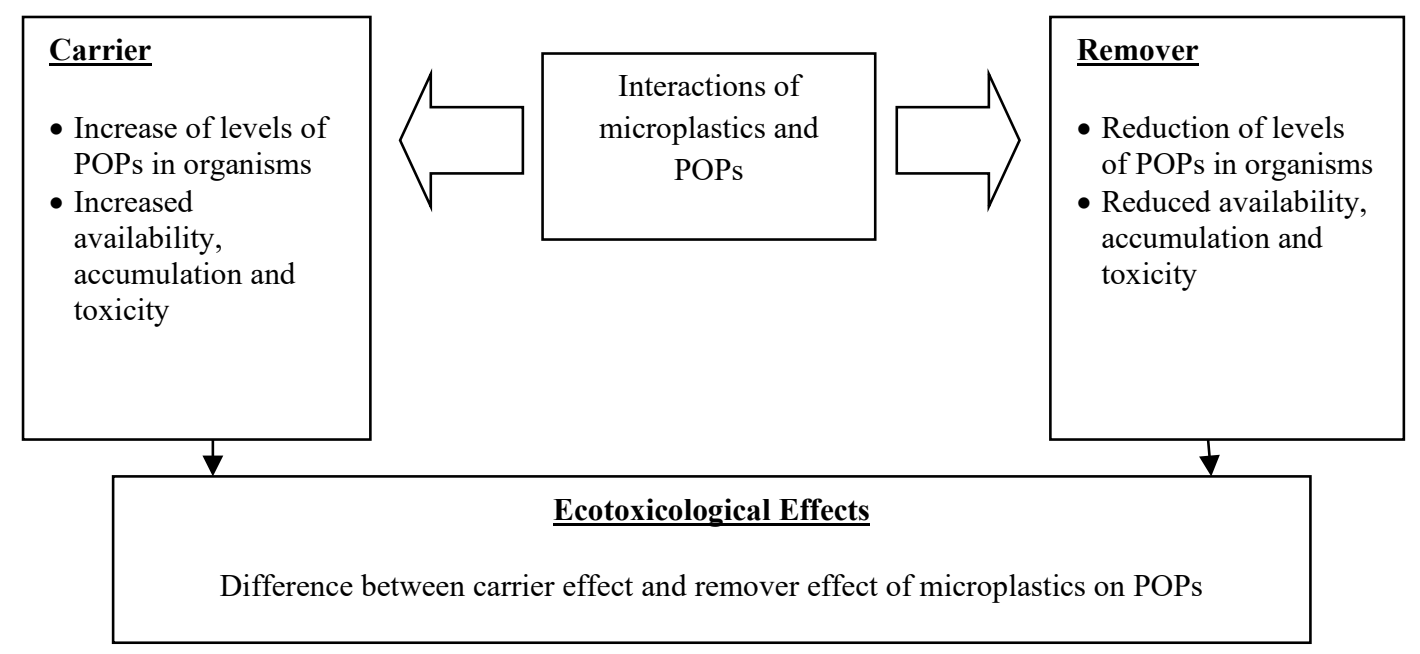

Figure 2. The POPs carrier and remover effects of microplastics determine their ecotoxicological effects

Nonetheless, the studies on microplastics as transporters of POPs are generally faced with the limitation that they have been conducted in laboratory at concentrations of microplastics substantially higher than in the environment. Besides, some studies are based on simulation. 
Therefore, it is questionable whether the findings of these studies could sufficiently represent how microplastics and the associated POPs actually behave in the environment [52]. Knowing that the sorption between SOPs and microplastics are affected by a multitude of factors, it leaves a huge gap to be filled. It is unclear how the sorbed POPs desorb and bioaccumulate under the single and concerted influences of different factors. For instance, studies related to how aged microplastics and microplastics of different types affect bioavailability and bioaccumulation of POPs, hence their toxicity, are comparatively less. In addition, current studies seem to be limited to a few types of POPs and organisms. There is a need to grow the current pool of knowledge on the interactions between microplastics and POPs, as well as their impacts of various components of the environment.

\section{Conclusion}

Microplastics have been a subject of heated discussion as more studies are pointing to their increasing presence in the environment. Their environmental concerns are aggravated by the increasing prevalence of POPs. Microplastics are known to leach chemicals and provide the surface for sorption of chemicals. Sorption of POPs to microplastics tend to concentrate the chemicals and this process has been deemed to alter or increase the toxicity and environmental risk of microplastics. This review highlights a generally low to insignificant impacts of POPssorbed microplastics on accumulation, bioavailability and toxicity of the experimented receptors, in contrary to the common assumption that a synergistic toxicity could result from such interaction. It also shows that more studies are needed to attest the ecotoxicological effects of POPs sorption to microplastics since the current studies are limited to only few types of common POPs and microplastics. It suggests that future studies could be extended to a wider range of environmentally significant POPs. Future studies could also employ a wide range of fresh, artificially aged and naturally aged microplastics, under different environmental conditions to examine their interactions with POPs and the ensuing ecological risks.

\section{Conflicts of Interest}

The authors declare no conflict of interest.

\section{References}

[1] Wong, J.K.H.; Lee, K.K.; Tang, K.H.D.; Yap, P-S. (2020). Microplastics in the freshwater and terrestrial environments: Prevalence, fates, impacts and sustainable solutions. Science of the Total Environment, 719, 137512.

[2] Tang, K.H.D. (2020). Effects of microplastics on agriculture: A mini-review. Asian Journal of Environment \& Ecology, 13(1):1-9.

[3] Ritchie, H.; Roser, M. (2018). Plastic pollution. Our World in Data. (assessed on 25 May 2021) Available online: https://ourworldindata.org/plastic-pollution?utm source=newsletter

[4] Lares, M.; Ncibi, M.C.; Sillanpää, M.; Sillanpää M. (2018). Occurrence, identification and removal of microplastic particles and fibers in conventional activated sludge process and advanced MBR technology. Water Research, 133, 236-46.

[5] Weinstein, J.E.; Crocker, B.K.; Gray, A.D. (2016). From macroplastic to microplastic: Degradation of high-density polyethylene, polypropylene, and polystyrene in a salt marsh habitat. Environmental Toxicology and Chemistry, 35(7), 1632-40. https://doi.org/10.1002/etc.3432

[6] Tang, K.H.D. (2020). Ecotoxicological impacts of micro and nanoplastics on marine fauna. Examines in Marine Biology and Oceanography, 3(2), 1-5.

[7] Alharbi, O.M.L.; Basheer, A.A.; Khattab, R.A.; Ali, I. (2018) Health and environmental effects of persistent organic pollutants. Journal of Molecular Liquids, 263, 442-53. 
[8] Wagner, M.; Lambert, S. (2018) Freshwater microplastics - The handbook of environmental Chemistry. (assessed 26 May 2021) Available online: http://www.springer.com/series/698

[9] Yu, F.; Yang, C.; Huang, G.; Zhou, T.; Zhao, Y.; Ma, J. (2020) Interfacial interaction between diverse microplastics and tetracycline by adsorption in an aqueous solution. Science of the Total Environment, 721, 137729.

[10] Guo, X.; Wang, J. (2019). Sorption of antibiotics onto aged microplastics in freshwater and seawater. Marine Pollution Bulletin, 149, 110511.

[11] Zhu, X.; Qiang, L.; Shi, H.; Cheng, J. (2020). Bioaccumulation of microplastics and its in vivo interactions with trace metals in edible oysters. Marine Pollution Bulletin, 154, 111079.

[12] Fred-Ahmadu, O.H.; Bhagwat, G.; Oluyoye, I.; Benson, N.U.; Ayejuyo, O.O.; Palanisami, T. (2020). Interaction of chemical contaminants with microplastics: Principles and perspectives. Science of the Total Environment, 706, 135978.

[13] Wang, F.; Wong, C.S.; Chen, D.; Lu, X.; Wang, F.; Zeng, E.Y. (2018). Interaction of toxic chemicals with microplastics: A critical review. Water Research, 139, 208-19.

[14] Liu, F.; Liu, G.; Zhu, Z.; Wang, S.; Zhao, F. (2019). Interactions between microplastics and phthalate esters as affected by microplastics characteristics and solution chemistry. Chemosphere, 214, 688-94.

[15] Menéndez-Pedriza, A.; Jaumot, J. (2020). Interaction of environmental pollutants with microplastics: A critical review of sorption factors, bioaccumulation and ecotoxicological effects. Toxics, 8(2), 40.

[16] Fu, L.; Li, J.; Wang, G.; Luan, Y.; Dai, W. (2021). Adsorption behavior of organic pollutants on microplastics. Ecotoxicology and Environmental Safety, 217, 112207.

[17] Tang, K.H.D.; Al Qahtani, H.M.S. (2020) Sustainability of oil palm plantations in Malaysia. Environment, Development and Sustainability, 22(6), 4999-5023.

[18] Tang, K.H.D. (2019). Phytoremediation of soil contaminated with petroleum hydrocarbons: A review of recent literature. Global Journal of Civil and Environmental Engineering, 1, 33-42.

[19] O'Connor, I.A.; Golsteijn, L.; Hendriks, A.J. (2016). Review of the partitioning of chemicals into different plastics: Consequences for the risk assessment of marine plastic debris. Marine Pollution Bulletin, 113(1), 17-24.

[20] Lee, K.K.; Tang, K.H.D. (2020). Agaricales (Gilled Mushrooms) as biosorbents of synthetic dye. Malaysian Journal of Medicine and Health Sciences, 16(SUPP11), 10-7.

[21] Hartmann, N.B.; Rist, S.; Bodin, J.; Jensen, L.H.S.; Schmidt, S.N.; Mayer, P. et al. (2017) Microplastics as vectors for environmental contaminants: Exploring sorption, desorption, and transfer to biota. Integrated Environmental Assessment and Management, 13(3), 488-93. https://doi.org/10.1002/ieam.1904

[22] Liu, L.; Fokkink, R.; Koelmans, A.A. (2016). Sorption of polycyclic aromatic hydrocarbons to polystyrene nanoplastic. Environmental Toxicology and Chemistry, 35(7), 1650-5. https://doi.org/10.1002/etc.3311

[23] Choong, W.S.; Hadibarata, T.; Tang, D.K.H. (2020). Abundance and distribution of microplastics in the water and riverbank sediment in Malaysia-A review. Biointerface Research in Applied Chemistry, 11(4), 11700-12.

[24] Wright, S.L.; Thompson, R.C.; Galloway, T.S. (2013). The physical impacts of microplastics on marine organisms: A review. Environmental Pollution, 178, 483-92.

[25] Ziccardi, L.M.; Edgington, A.; Hentz, K.; Kulacki, K.J.; Kane Driscoll, S. (2016). Microplastics as vectors for bioaccumulation of hydrophobic organic chemicals in the marine environment: A state-of-the-science review. Environmental Toxicology Chemistry, 35(7), 1667-76. https://doi.org/10.1002/etc.3461

[26] Bakir, A.; Rowland, S.J.; Thompson, R.C. (2014). Enhanced desorption of persistent organic pollutants from microplastics under simulated physiological conditions. Environmental Pollution, $185,16-23$.

[27] Velzeboer, I.; Kwadijk, C.J.A.F.; Koelmans, A.A. (2014). Strong sorption of PCBs to nanoplastics, microplastics, carbon nanotubes, and fullerenes. Environmental Science \& Technology, 48(9), 4869-76. https://doi.org/10.1021/es405721v.

[28] Jahnke, A.; Arp, H.P.H.; Escher, B.I.; Gewert, B.; Gorokhova, E.; Kühnel, D. et al. (2017). Reducing uncertainty and confronting ignorance about the possible impacts of weathering plastic in the marine environment. Environmental Science \& Technology Letters, 4(3), 85-90. https://doi.org/10.1021/acs.estlett.7b00008. 
[29] Andrady, A.L. (2017). The plastic in microplastics: A review. Marine Pollution Bulletin, 119(1), $12-22$.

[30] Fotopoulou, K.N.; Vakros, J.; Karapanagioti, H.K. (2014). Surface properties of marine microplastics that affect their interaction with pollutants and microbes. In CIESM Workshop Monographs, pp. 377-81.

[31] Frias, J.P.G.L.; Sobral, P.; Ferreira, A.M. (2010). Organic pollutants in microplastics from two beaches of the Portuguese coast. Marine Pollution Bulletin, 60(11), 1988-92.

[32] Fisner, M.; Majer, A.; Taniguchi, S.; Bícego, M.; Turra, A.; Gorman, D. (2017). Colour spectrum and resin-type determine the concentration and composition of Polycyclic Aromatic Hydrocarbons (PAHs) in plastic pellets. Marine Pollution Bulletin, 122(1), 323-30.

[33] Müller, A.; Becker, R.; Dorgerloh, U.; Simon, F-G.; Braun, U. (2018). The effect of polymer aging on the uptake of fuel aromatics and ethers by microplastics. Environmental Pollution, 240, 63946.

[34] Syberg, K.; Khan, F.R.; Selck, H.; Palmqvist, A.; Banta, G.T.; Daley, J. et al. Microplastics: addressing ecological risk through lessons learned. Environmental Toxicology and Chemistry, 34(5), 945-53. https://doi.org/10.1002/etc.2914.

[35] Lee, H.; Shim, W.J.; Kwon, J-H. (2014). Sorption capacity of plastic debris for hydrophobic organic chemicals. Science of the Total Environment, 470-471, 1545-52.

[36] Rochman, C.M.; Hoh, E.; Hentschel, B.T.; Kaye, S. (2013). Long-term field measurement of sorption of organic contaminants to five types of plastic pellets: Implications for plastic marine debris. Environmental Science \& Technology, 47(3), 1646-54. https://doi.org/10.1021/es303700s

[37] Hüffer, T.; Hofmann, T. (2016). Sorption of non-polar organic compounds by micro-sized plastic particles in aqueous solution. Environmental Pollution, 214, 194-201.

[38] Teuten, E.L.; Saquing, J.M.; Knappe, D.R.U.; Barlaz, M.A.; Jonsson, S.; Björn, A. et al. (2009). Transport and release of chemicals from plastics to the environment and to wildlife. Philosophical Transactions of the Royal Society B, 364(1526), 2027-45. https://doi.org/10.1098/rstb.2008.0284

[39] Endo, S.; Yuyama, M.; Takada, H. (2013). Desorption kinetics of hydrophobic organic contaminants from marine plastic pellets. Marine Pollution Bulletin, 74(1), 125-31.

[40] Kärrman, A.; Schönlau, C.; Engwall, M. (2016). Exposure and effects of microplastics on wildlife: A review of existing data. (accessed on 25 May 2021) Available online: https://www.divaportal.org/smash/get/diva2:921211/FULLTEXT01.pdf

[41] Avio,C.G.; Gorbi, S.; Milan, M.; Benedetti, M.; Fattorini, D.; d'Errico, G. et al. (2015). Pollutants bioavailability and toxicological risk from microplastics to marine mussels. Environmental Pollution, 198, 211-22.

[42] Tang, K.H.D. (2020). A comparative overview of the primary Southeast Asian safety and health laws. International Journal of Workplace Health Management, 13(6), 601-632. https://doi.org/10.1108/IJWHM-10-2019-0132.

[43] Diepens, N.J.; Koelmans, A.A. (2018). Accumulation of plastic debris and associated contaminants in aquatic food webs. Environmental Science \& Technology, 52(15):8510-20. https://doi.org/10.1021/acs.est.8b02515.

[44] Kleinteich, J.; Seidensticker, S.; Marggrander, N.; Zarfl, C. (2018). Microplastics reduce shortterm effects of environmental contaminants. Part II: Polyethylene particles decrease the effect of polycyclic aromatic hydrocarbons on microorganisms. International Journal of Environmental Research and Public Health, 15(2), 287.

[45] Pittura, L.; Avio, C.G.; Giuliani, M.E.; d'Errico, G.; Keiter, S.H.; Cormier, B. et al. (2018). Microplastics as vehicles of environmental PAHs to marine organisms: Combined chemical and physical hazards to the Mediterranean Mussels, Mytilus galloprovincialis. Frontiers in Marine Science, 5, 103. https://www.frontiersin.org/article/10.3389/fmars.2018.00103.

[46] Magara, G.; Elia, A.C.; Syberg, K.; Khan, F.R. (2018). Single contaminant and combined exposures of polyethylene microplastics and fluoranthene: accumulation and oxidative stress response in the blue mussel, Mytilus edulis. Journal of Toxicology and Environmental Health Part A, 81(16), 761-73. https://doi.org/10.1080/15287394.2018.1488639.

[47] Batel, A.; Borchert, F.; Reinwald, H.; Erdinger, L.; Braunbeck, T. (2018). Microplastic accumulation patterns and transfer of benzo[a]pyrene to adult zebrafish (Danio rerio) gills and zebrafish embryos. Environmental Pollution, 235, 918-30.

[48] Grigorakis, S.; Drouillard, K.G. (2018). Effect of microplastic amendment to food on diet assimilation efficiencies of PCBs by fish. Environmental Science \& Technology, 52(18), 10796- 
802. https://doi.org/10.1021/acs.est.8b02497.

[49] Devriese, L.I.; De Witte, B.; Vethaak, A.D.; Hostens, K.; Leslie, H.A. (2017). Bioaccumulation of PCBs from microplastics in Norway lobster (Nephrops norvegicus): An experimental study. Chemosphere, 186, 10-6.

[50] Scopetani, C.; Cincinelli, A.; Martellini, T.; Lombardini, E.; Ciofini, A.; Fortunati, A. et al. Ingested microplastic as a two-way transporter for PBDEs in Talitrus saltator. Environmental Research, 167, 411-7.

[51] Pannetier, P.; Cachot, J.; Clérandeau, C.; Faure, F.; Van Arkel, K.; de Alencastro, L.F. et al. (2019). Toxicity assessment of pollutants sorbed on environmental sample microplastics collected on beaches: Part I-adverse effects on fish cell line. Environmental Pollution, 248, 1088-97.

[52] da Costa, J.P.; Santos, P.S.M.; Duarte, A.C.; Rocha-Santos, T. (2016). (Nano)plastics in the environment - Sources, fates and effects. Science of the Total Environment, 566-567, 15-26.

(C) 2021 by the authors. This article is an open access article distributed under the terms and conditions of the Creative Commons Attribution (CC BY) license (http://creativecommons.org/licenses/by/4.0/). 